\title{
Microstructure of nanostructured amorphous Si-Au-alloys
}

\author{
A. STURM and A. WIEDENMANN
}

Hahn-Meitner-Institut Berlin GmbH, AG N5, Glienicker Str. 100, 1000 Berlin 39, Germany

\begin{abstract}
Small Angle Neutron Scattering (SANS) was employed to characterize the microstructure of nanostructured amorphous $\mathrm{Si}_{1-\mathrm{X}} \mathrm{Au}_{\mathrm{X}}$-alloys $(0.1<\mathrm{x}<0.3)$ produced by an inert-gas condensation and in-situ consolidation technique. Amorphous grains are embedded in a matrix of lower density, formed by grain boundaries and free volumes. The relative densities were found to be dependent on the average particle sizes and volume fractions, but independent of the alloy composition. In all samples the SANS intensity decreases according to a power law $\sim Q^{-3.37}$ at low $Q$, which is attributed to a surface fractal structure of dimension $\mathrm{D}_{\mathrm{S}}=2.63$.
\end{abstract}

\section{INTRODUCTION}

Nanostructured amorphous (na-) Si-rich Si-Au-alloys have been produced by an inert gas condensation process followed by in-situ consolidation of the amorphous grains with several nanometer diameter. The interest in these nanostructured amorphous solids arises from the conception that on compacting the isolated amorphous grains, a solid with a high density of topological defects is created. These topological defects should be characterized by a reduction in the short range order of the atomic arrangements and in the atomic density, similar to the situation found in shear bands in metallic glasses $[1,2]$.

In this paper we present the microstructural characterisation as obtained by SANS and density measurements.

\section{SAMPLE PREPARATION}

The amorphous samples are produced by the same method used to produce nanostructured crystalline solids [3]. Au and $\mathrm{Si}$ are evaporated from the molten alloy into a $3 \mathrm{mbar} \mathrm{He}$ atmosphere. In the vicinity of the evaporation source, nanometer-sized particles grow by homogeneous nucleation and coalescence. The particles are transported via convective flow of the He-gas to a liquid nitrogen cooled surface where they loosely adhere and are scraped off. When enough powder has accumulated to form a sample, evaporation is stopped, high vacuum is re-established and the powder is consolidated in high vacuum at $1.6 \times 10^{-9}$ Pa during $120 \mathrm{~s}$.

The samples are discs of $8 \mathrm{~mm}$ diameter and typically 150-500 $\mu \mathrm{m}$ thickness. The composition of the samples varies by \pm 1 at\% across the surface, as determined by energy-dispersive X-ray fluorescence measurements. The amorphous state was proofed by X-ray [4] and neutron wide angle scattering [5]. 


\section{DENSITY MEASUREMENTS}

The effective density $\rho_{E}$ (including only closed porosity) was determined by the Archimedes method in Diethyphthalat $\mathrm{C}_{12} \mathrm{H}_{14} \mathrm{O}_{4}$ according to $\rho_{\mathrm{E}}=\mathrm{m}_{\mathrm{A}} \rho_{\mathrm{D}} /\left(\mathrm{m}_{\mathrm{A}}-\mathrm{m}_{\mathrm{L}}\right)$, where $\mathrm{m}_{\mathrm{A}}$ is the weight of the sample in air and $\mathrm{m}_{\mathrm{L}}$ is the weight of the sucked sample in Diethyphthalat with the density $\rho_{D}$. To make sure that all open porosity was filled with liquid the samples have been kept for 3 days in Diethyphthalat before measuring $\mathrm{m}_{\mathrm{L}}$. Closed porosity is the part of the free volume which can not be filled with liquid.

In Fig. 1a the effective densities $\rho_{E}$ of the na-samples are shown as a function of the Au-content. They are compared with density values of amorphous $\mathrm{Si}-\mathrm{Au}$ films produced by electron beam evaporation onto $77 \mathrm{~K}$ substrates [6], which are consindered to be homogeneous and to have maximum density. The density of the na-samples increases with increasing Au-content, but compared to the density of the amorphous films the corresponding values of the na-samples are significantly lower and strongly spreaded. This is shown in Fig. $1 \mathrm{~b}$, where the relative density, i.e. the ratio of the effective density to the film density of the same Au-content, is plotted. The relative densities fluctuate between $62 \%$ and $88 \%$, while no significant correlation with the Au-content can be deduced.

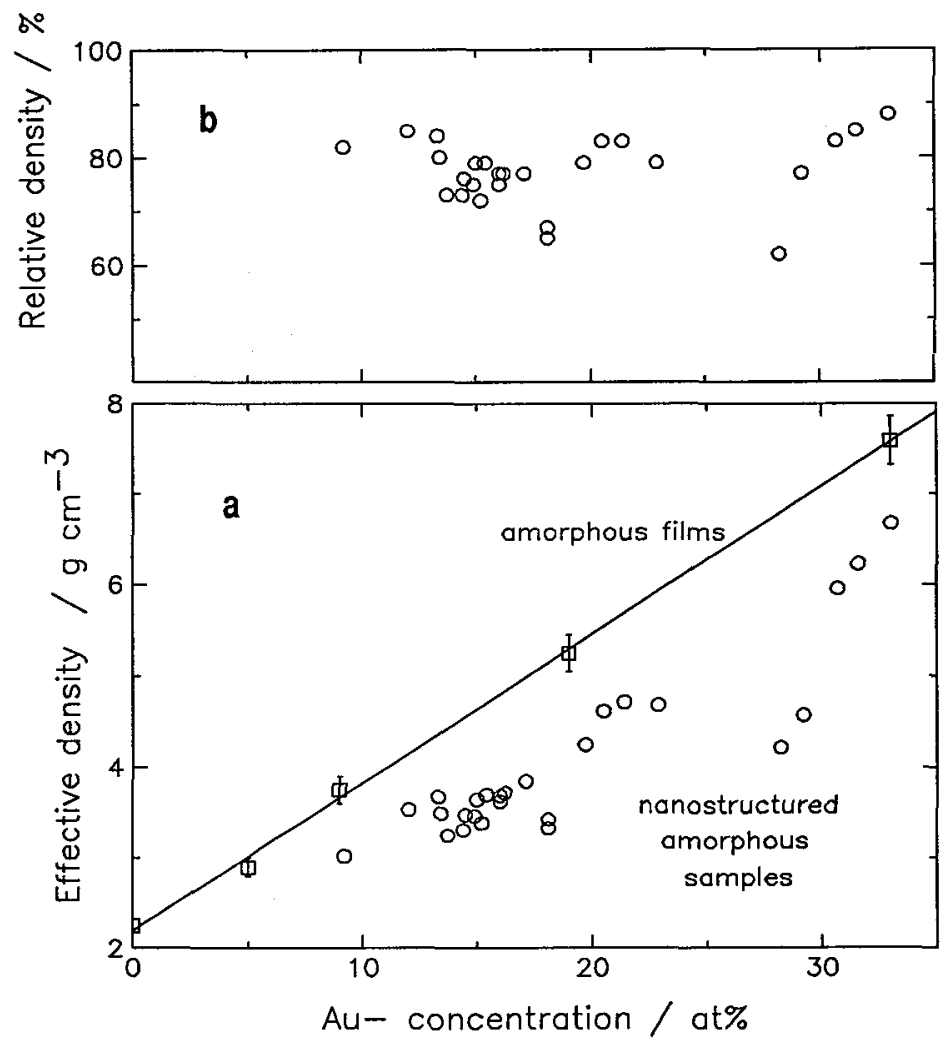

Fig.1 a: Effective densities (including only closed porosity) of the na-samples and density of amorphous films; $\quad$ b: Relative densities, i.e. the ratio of the effective density to the film density of the same $\mathrm{Au}-$ content, plotted versus $\mathrm{Au}$-concentration. 
The SANS measurements were carried out at the small-angle cameras D17 and D11 at the High Flux Reactor at the ILL Grenoble. A range of the momentum transfer $Q$ from $0,01 \mathrm{~nm}^{-1}$ to $3.6 \mathrm{~nm}^{-1}$ was covered $[Q=(4 \pi / \lambda) \sin \theta, 2 \theta=$ scattering angle]. All measurements were performed at room temperature, with the specimen in air. The intensity data were corrected for background scattering, transmission, detector efficency and electronic noise. Absolute values of the differential scattering cross-section $\mathrm{d} \sigma(\mathrm{Q}) / \mathrm{d} \Omega$ in units of barn/(sr at.) were determined by comparision with the incoherent scattering power of a standard water specimen. The two-dimensional scattering cross-sections recorded were always of perfect radial symmetry around the central beam, and could consequently be averaged for equal radial positions of the detector.

Fig. 2 is a double logarithmic presentation of the scattering cross-section in absolute units for samples with different relative densities, as a function of the momentum transfer $\mathrm{Q}$. For the sample with the highest relative density of $88 \%$ a power law of the scattering cross section $d \sigma(\mathrm{Q}) / \mathrm{d} \Omega \sim \mathrm{Q}^{-\alpha}$ over almost two orders of magnitude in $\mathrm{Q}$ and five orders of magnitude in intensity was observed below $\mathrm{Q}=1 \mathrm{~nm}^{-1}$. The exponent $\alpha$ of the power law was 3.37. For all other samples an additional scattering contribution occurs overlapping the power law in the medium q-range. For samples with decreasing relative density, the intensity of the scattering contributions in the medium q-range increases, while at low $Q$ the intensity decreases.

A quantitative evolution of the SANS data was carried out by calculating $d \sigma(Q) / d \Omega$ on the basis of a modelled microstructure:

- large inhomogeneities, characterized by a $Q^{-\alpha}$ power law scattering at small $Q$, with $\alpha=3.37$ for all samples;

- amorphous grains, surrounded by a matrix of reduced density, consisting of grain boundaries and free volumes ; the grains were assumed to have a spherical shape and a log-normal size distribution;

- incoherent background scattering.

This modelled structure function could be fitted by means of an iterative trial and error procedure to the scattering curves of all samples.

The average radius of the log-normal size distribution is directly obtained from the fit and plotted in Fig. 3, versus the relative density. In samples with increasing relative density, a decreasing particle size is observed. When only density fluctuations between grains and the surrounding boundaries are assumed, further sample parameters can be determined from the intensity parameter of the fit and the effective density. It turned out that, for increasing relative density from $65 \%$ to $88 \%$ the volume fraction of the grains increased from 30 to 60 vol\%, while the fraction of free volumes decreased from to 30 to 0 vol\%. The density of the grain boundaries is about $80 \%$ of the grain density and almost independent of the relative density of the na-sample [5].

\section{SUMMARY}

Nanostructured amorphous Si-Au-alloys were produced by compacting precursor isolated amorphous grains. From SANS a correlation of smaller grain sizes with higher relativ densities was found. While all samples were compacted with the same external parameters, higher densities are achieved with smaller grains because grain rearrangement and sliding is the dominating compaction process [7]. The observed scattering according to a power law $\sim Q^{-3.37}$ is attributed to the self similar nature of density fluctuations of large size: the compaction of the grains to na-samples gives rise to a surface fractal structure which is characterized by a fractal dimension $\mathrm{D}_{\mathrm{S}}=2.63$. 
[1] MASUMOTO T., KIMURA H., INOUE A. and WASEDA Y., Mat. Sci. and Eng. 23(1976)141.

[2] DONOVAN P.E. and STOBBS W.M., Acta Met. 29(1981)1419.

[3] GLEITER H., Prog. Mater. Sci. 33(1989)223.

[4] WEISSMÜLLER J., Phys. Lett. A 145(1990)130; J. Non-Cryst. Solids 142(1992)70.

[5] STURM A., Thesis, TU Berlin D83, (1993).

[6] MANGIN Ph., MARCHAL G., MOUREY C. and JANOT Chr., Phys. Rev. B 21(1980)3047.

[7] GERMAN R.D., Powder Metallurgy Science, MPIF, Princeton, NJ (1984).

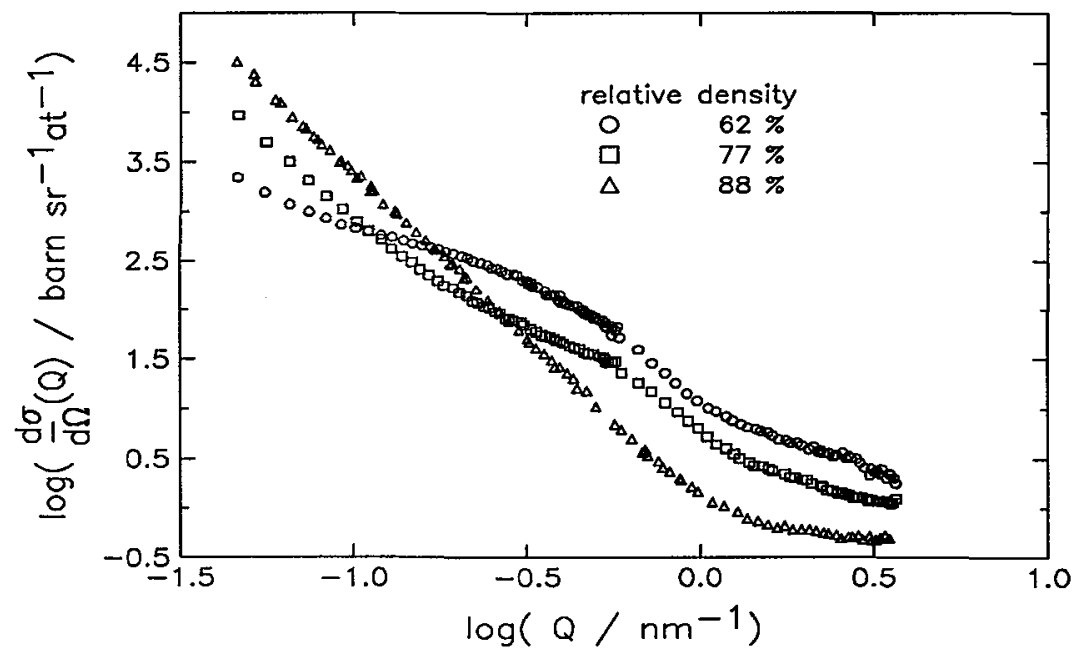

Fig.2 Double-logarithmic plot of SANS intensity data of na-Si-Au-samples with different relative densities, versus momentum transfer $Q$.

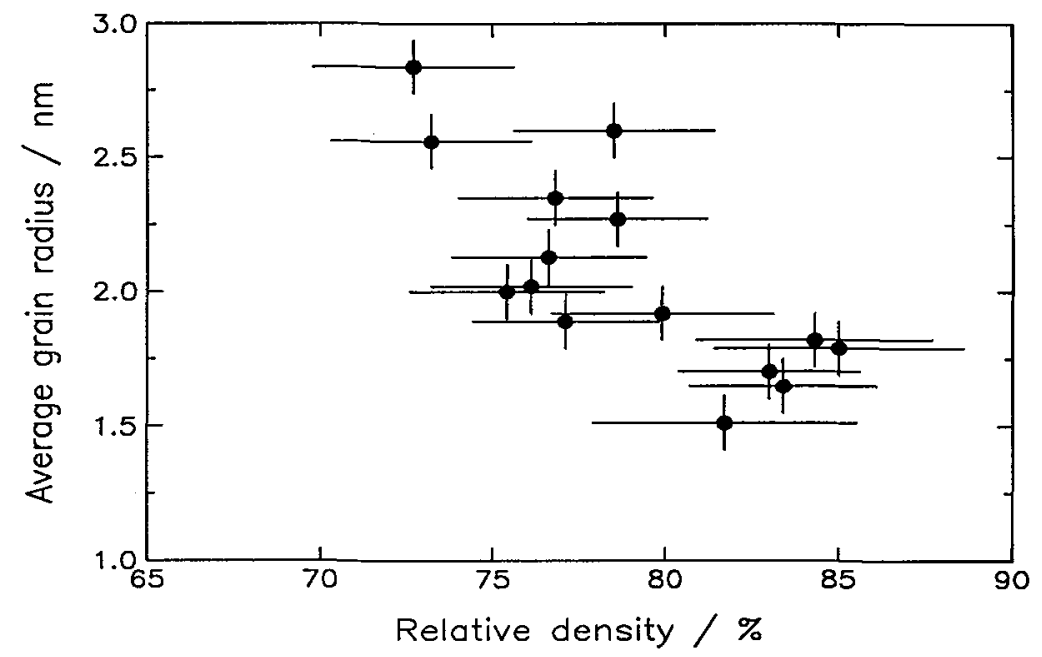

Fig. 3 Average grain radius as determined from the model fit, plotted versus the relative density of the nanostructured amorphous samples. 